\title{
NMR parameters of FNNF as a test for coupled-cluster methods: CCSDT shielding and
} CC3 spin-spin coupling

\author{
Jaszuski, Micha; Sauer, Stephan P A; Faber, Rasmus; Wilson, David J D
}

Published in:

Physical Chemistry Chemical Physics

Link to article, DOI:

$10.1039 / \mathrm{d} 0 \mathrm{cp} 02730 \mathrm{~h}$

Publication date:

2020

Document Version

Peer reviewed version

Link back to DTU Orbit

Citation (APA):

Jaszuski, M., Sauer, S. P. A., Faber, R., \& Wilson, D. J. D. (2020). NMR parameters of FNNF as a test for coupled-cluster methods: CCSDT shielding and CC3 spin-spin coupling. Physical Chemistry Chemical Physics, 22, 21350-21359. https://doi.org/10.1039/d0cp02730h

\section{General rights}

Copyright and moral rights for the publications made accessible in the public portal are retained by the authors and/or other copyright owners and it is a condition of accessing publications that users recognise and abide by the legal requirements associated with these rights.

- Users may download and print one copy of any publication from the public portal for the purpose of private study or research.

- You may not further distribute the material or use it for any profit-making activity or commercial gain

- You may freely distribute the URL identifying the publication in the public portal 


\title{
Journal Name
}

\section{ARTICLE TYPE}

Cite this: DOI: $00.0000 / \operatorname{xxxxxxxxxx}$

\section{NMR parameters of FNNF as a test for coupled- cluster methods: CCSDT shielding and CC3 spin-spin coupling}

\author{
Michał Jaszuński, ${ }^{a}$ Stephan P. A. Sauer, ${ }^{b}$ Rasmus Faber, ${ }^{c}$ and David J. D. Wilson ${ }^{d}$
}

Received Date

Accepted Date

DOI: $00.0000 / x x x x x x x x x x$

\begin{abstract}
NMR shielding and spin-spin coupling constants of cis and trans isomers of FNNF have been determined to near-quantitative accuracy from ab initio calculations. The FNNF system, containing multiple N-F bonds and fluorine atoms, provides a severe test of computational methods. Coupled-cluster methods were used with large basis sets and complete basis set (CBS) extrapolations of the equilibrium geometry results, with vibrational and relativistic corrections. Shielding constants were calculated with basis sets as large as aug-cc-pCV7Z, together with coupled-cluster expansions up to CCSDT, at the all-electron CCSD(T)/aug-cc-pCVQZ optimized geometries. Spin-spin coupling constants have been determined with specialized versions of the correlation consistent basis sets ccJ-pVXZ, further augmented with diffuse functions. All-electron coupled-cluster methods up to CC3 were applied in these calculations. The results of this work highlight the application of state-of-the-art theoretical techniques, and provide the most accurate NMR properties of FNNF to date, which can serve to guide and supplement NMR experimentation.
\end{abstract}

\section{Introduction}

Nuclear magnetic resonance (NMR) is one of the most useful tools for molecular structure determination. NMR shielding and spin-spin coupling constants are extremely sensitive to nuclear environment, which enables understanding of molecular structure. Developments in computational quantum chemistry methods now enable the accurate calculation of NMR properties, including absolute shieldings that are not directly measurable from experiment. ${ }^{1+-3]}$ High-level theoretical calculations are now capable of producing NMR parameters with accuracy that is comparable to experiment. As such, there is current interest in performing quantum mechanical calculations of shielding and spin-spin coupling constants for molecules that are difficult to investigate experimentally in order to validate and supplement experimental data. 4

Of particular interest is the accurate prediction of ${ }^{19} \mathrm{~F}$ NMR data. Although many computational studies have been presented recently, $\frac{5-16}{16}$ the determination of molecular magnetic properties

\footnotetext{
${ }^{a}$ Institute of Organic Chemistry, Polish Academy of Sciences, Kasprzaka 44-52, 01-224 Warsaw, Poland

${ }^{b}$ Department of Chemistry, University of Copenhagen, Copenhagen $\emptyset$, Denmark ${ }^{c}$ Department of Chemistry, Technical University of Denmark, Kgs. Lyngby, Denmark ${ }^{d}$ Department of Chemistry and Physics, La Trobe Institute for Molecular Science, La Trobe University, Melbourne, Victoria 3086, Australia. E-mail: david.wilson@latrobe.edu.au
}

of fluorine-containing molecules is still challenging for theoretical methods. ${ }^{17}$ Molecules containing N-F bonds are particularly challenging, in part due to large anharmonic vibrational effects. $\frac{15}{[1}$ In this work we have investigated the NMR shielding and spin-spin coupling of FNNF (dinitrogen difluoride, $\mathrm{N}_{2} \mathrm{~F}_{2}$ ), which represents an extreme test of quantum chemical methods. FNNF can exist as either a cis or trans conformer, and therefore provides two similar yet different molecular environments to model. Multiconfiguration self-consistent field (MCSCF) calculations of both shielding and spin-spin coupling constants in cis- and trans-FNNF have been described by Jaszuński et al. in 1996, 18 and more recently Faber and Sauer reported coupled-cluster singles and doubles (CCSD) studies of the coupling. ${ }^{19}$ However, with advancements in coupled-cluster (CC) methods for the use of magnetic property calculations, particularly the availability of important triple excitations via CC3 or CCSDT, as well as a greater understanding of necessary contributions to calculated properties, a reinvestigation of the magnetic properties of FNNF is warranted.

Theoretical studies of NMR shielding constants are challenging due to the necessary requirements for accurate results. Careful consideration of electron correlation effects is important; CC methods have become the standard for NMR property calculations, with most recent studies employing at least CC singles, doubles, and perturbative triples CCSD(T). Additionally, core correlation is important for accurate shielding constants and so allelectron calculations are required. ${ }^{19} \mathrm{~F}$ NMR calculations are addi- 
tionally challenging due to the large number of lone pairs. Gaugeindependence must also be accounted for through the usage of gauge-including atomic orbitals (GIAOs), which are available for CC methods. 20.28 Furthermore, accurate comparison with experiment requires consideration of zero-point vibrational effects (see e.g. refs 9 12 29.-33 and the recent review 34). Relativistic effects have also been shown to be non-negligible even for light atoms. $35 \sqrt{36}$

Here we have employed CC methods up to CCSDT for the calculation of NMR shielding properties and up to CC3 for the spinspin coupling constants for both isomers of FNNF. A benefit of the coupled-cluster wave function approach is that the hierarchy of CC methods can be systematically expanded. In this work we have explored the convergence of NMR constants as a function of both the basis set and coupled-cluster expansion. For the calculation of molecular properties, the effect of truncations of the wave function expansion and basis sets in the calculation of NMR properties have been explored for both ab initio and densityfunctional theory (DFT) methods. $\frac{37.41}{4}$ Extrapolation enables an estimate of the complete-basis set (CBS) limit to be calculated. Kupka et al. $33 \mid 42,46$ have illustrated this approach with HartreeFock (HF), DFT, MP2, and coupled-cluster methods. We have previously demonstrated the efficacy of this approach in the calculation of NMR shielding constants of $\mathrm{NF}_{3}, \mathrm{PF}_{3}$, and $\mathrm{AsF}_{3}$. 15

Despite the close relationship between NMR shieldings and spin-spin coupling constants, the challenges that must be addressed in order to compute them accurately are different. On one hand the local nature of all perturbations means that spinspin coupling constants are not affected by gauge-origin problems. However, the triplet spin-symmetry of some of the perturbing operators means that the employed electronic structure method should yield a ground state stable with respect to such perturbations. It is well known that HF tends to severely underestimate the energy gap between the lowest singlet and triplet state, and as a result HF values for spin-spin coupling constants are often qualitatively incorrect (see e.g. refs 47-51). The HF ground state is used as a reference in CC theory, so if the coupling constants are calculated directly as the derivatives of the CC energy the results are still potentially unstable. $\frac{52}{}$ A stable procedure for the calculation of spin-spin coupling constants in standard CC theory can be obtained by the so-called "unrelaxed derivative" approach where the HF state is taken as a parameter independent of any perturbations. 52 Unrelaxed CCSD values for spin-spin coupling constants have been successfully computed for numerous molecules; we shall make use here of the pertinent cisand trans-FNNF results. 19

However, in order to obtain very accurate results the inclusion of triple excitations is needed and the unrelaxed approach is not, at the theoretical level, compatible with the perturbation analysis leading to the CCSD(T) approach. Using the CC3 method has been reported as an alternative to go beyond CCSD. 14/53 While CC3 is an iterative approximate method and thus fundamentally more costly than $\operatorname{CCSD}(\mathrm{T})$, it is still significantly faster than full CCSDT calculations. Furthermore, it has previously been shown that CC3 recovers the majority of the triples contribution to fluorine spin-spin coupling constants. 14
We employ in this work a standard approach beginning with calculation of the non-relativistic shielding and spin-spin coupling at an optimized geometry. The non-relativistic properties are augmented with vibrational and relativistic corrections to obtain a relativistic vibrationally-averaged property. The current approach that combines optimized equilibrium geometry coupled-cluster values including triple excitations computed with large basis sets together with corrections for vibrational averaging and relativistic effects can be expected to provide the most accurate theoretical values to date for the NMR shielding and spin-spin coupling constants in FNNF.

\section{Computational Details}

All coupled-cluster calculations have been performed using the CFOUR program ${ }^{54}$ and MRCC program. ${ }^{55[56}$ Studies of the relativistic effects on the isomerization energy, NMR shielding and spin-spin coupling at the Dirac-Hartree-Fock and Dirac-KohnSham level were carried out with the ReSpect 5.1.0 program. 57

The geometries of cis- and trans-FNNF employed in this work are all-electron $\operatorname{CCSD}(\mathrm{T})$ results with the aug-cc-pCVQZ basis set, taken from ref 19. Harmonic vibrational frequencies were calculated at the same level of theory in CFOUR. ${ }^{54}$ To quantify the cistrans isomerization energy, electronic energies were calculated up to the CCSDTQ level of theory in CFOUR and MRCC. $55 \mid 56$

The calculation of absolute shielding constants employed the augmented core-valence correlation consistent basis sets (aug-ccpCVXZ with $X=D, T, Q, 5,6$, and 7). 58.64 In the following these basis sets are abbreviated as ACVXZ. The ACV7Z sets for $\mathrm{N}$ and $\mathrm{F}$ were built as part of the current work. Extrapolation to the complete basis set (CBS) employed the same approach described previously. ${ }^{15}$ For the indirect nuclear spin-spin coupling constants, the specialized coupling constant versions of the correlation consistent basis sets ccJ-pVXZ, ${ }^{65}$ were employed, which were further augmented with the diffuse functions of the aug-cc-pVXZ basis sets. 19 These basis sets are denoted AVXZ-J and should not be confused with the similarly labeled aug-cc-pVTZ-J basis sets. $.5,50$ In some calculations the AVXZ-J basis sets were used in a completely uncontracted form, denoted as AVXZ-Juc.

The CCSD results quoted below for the spin-spin coupling constants at the optimized equilibrium geometry are taken from ref 19. whereas the CC3 results as well as the vibrational corrections to the coupling constants were calculated for this work.

For the analysis of the importance of vibrational corrections to NMR properties we used the VPT2 approach $34 \sqrt{66}$ as implemented $^{30}$ in CFOUR. In the calculations of the vibrational corrections to the shielding constants at the $\operatorname{CCSD}(\mathrm{T})$ level different combinations of basis sets were employed. In addition to the ACVTZ both for the force constants and shielding constant derivatives vibrational corrections were calculated with tz2p and qz2p basis sets. 30 Separate basis sets were considered for the cubic force field calculation and NMR shielding derivative calculation.

For the calculation of the vibrational corrections to the coupling constants at the CCSD level two different combinations of basis sets were employed. In the first version both the harmonic and anharmonic force constants and the coupling constants were obtained with the same basis set: aug-cc-pVQZ for the Fermi contact 
(FC), para- and diamagnetic spin-orbit (PSO and DSO) contributions and aug-cc-pVTZ for the spin-dipole (SD) contributions. In the second version the force constants were calculated with the aug-cc-pVQZ basis set, while the derivatives of the coupling constant surfaces were calculated with the aug-ccJ-pVTZ basis sets for the FC, PSO and DSO contributions and the aug-ccJ-pVDZ basis set for the SD contributions.

All results are calculated and given for ${ }^{15} \mathrm{~N}$ species.

\section{Results and discussion}

\subsection{Geometry}

Comparison with experimental data and previous high-level calculations enables an assessment of the accuracy of the optimized geometry. There are two experimental studies for the geometry of FNNF; a microwave (MW) study from 1963 that only reported the geometry of the cis isomer, 67 and an electron-diffraction (ED) study from 1967 that reported geometries of both the cis and trans isomers. $\underline{68}$ Experimental and calculated geometric parameters are given in Table 1

For cis-FNNF there is excellent agreement between the MW and ED structures for $\mathrm{r}(\mathrm{NN})$ and $\angle(\mathrm{NNF})$, with the all-electron CCSD(T)/ACVQZ results in excellent agreement with the experimental parameters. However, for $\mathrm{r}(\mathrm{NF})$ the ED structure yields a significantly longer bond distance (1.410 ̊) compared to the MW structure (1.384 $\AA$ ). Previously reported frozen-core MP2/TZ2Pf ${ }^{69}$ and CCSD(T)/AVTZ ${ }^{70}$ results (Table 1) are in good agreement with our all-electron $\operatorname{CCSD}(\mathrm{T})$ results, although the frozen-core MP2 and $\operatorname{CCSD}(\mathrm{T})$ calculated bond distances are consistently greater than our all-electron $\operatorname{CCSD}(\mathrm{T})$ values. The allelectron CCSD(T)/ACVQZ optimized bond distance lies within the error bars of the MW-derived bond distance, but lies outside the lower boundary of error bars of the ED value. To assess basis set convergence a geometry optimization was carried out at the all-electron CCSD(T) level of theory with the ACV5Z basis set, which indicated that the ACVQZ geometries are converged within $0.002 \AA$, while as expected, expansion of the basis set leads to successively shorter bond distances. From the excellent agreement of the all-electron $\operatorname{CCSD}(\mathrm{T})$ results and the MW geometry it is concluded that the ED determined r(NF) bond distance is too long, in agreement with Christe et al. $\frac{70}{70}$ Moreover, it is suggested that the true value lies at the lower bound of the MW error bar.

For the trans isomer, where no MW structure is available, it is again noted that the CCSD(T)/ACVQZ calculated N-F bond distance is significantly shorter and lies outside the error bars of the ED value. Extending our analysis of the cis geometry results, we can similarly expect that the ED-derived N-F bond distance is too long. Again, the frozen-core MP2/TZ2Pf 69 and $\operatorname{CCSD}(\mathrm{T}) / \mathrm{AVTZ}^{70}$ bond distances are consistently greater than our all-electron CCSD(T)/ACVQZ results. We conclude that the all-electron CCSD(T)/ACVQZ calculated geometries may be considered converged and appropriate for a study of molecular properties of FNNF. The CCSD(T)/ACV5Z geometries may be considered the most accurate reported to date.

\subsection{Cis-Trans Isomerization Energy}

The high-level calculations reported here enable an examination of the cis-trans isomerization energy of FNNF, with results collated in Table 2 At the CCSD/AVTZ level of theory the T1 diagnostic is calculated to be 0.018 (cis) and 0.016 (trans), which are both below 0.02 and indicative that both isomers can be reasonably treated with a single reference method. 71

At the HF-SCF level the trans isomer is favoured by 6.23 $\mathrm{kJ} / \mathrm{mol}$, however correlation energy favours the cis isomer and is required to provide a reliable isomerization energy. Single and double excitations with all-electron CCSD $(\Delta \mathrm{SD})$ favours cis by $3.55 \mathrm{~kJ} / \mathrm{mol}$, while perturbative triples with $\operatorname{CCSD}(\mathrm{T})$ favours cis by a further $8.49 \mathrm{~kJ} / \mathrm{mol}$.

Our CBS results indicate that the cis isomer is more stable by $5.56 \mathrm{~kJ} / \mathrm{mol}$, whereas if we employ the experimental $\Delta \mathrm{ZPVE}$ of $-0.473 \mathrm{~kJ} / \mathrm{mol}$ we obtain a CBS isomerization of $5.72 \mathrm{~kJ} / \mathrm{mol}$. These values are marginally lower than $5.9 \mathrm{~kJ} / \mathrm{mol}$ calculated by Christe et al.70 at the CCSD(T)/AVTZ level of theory, and lower than the MP2/TZ2Pf value of $7.9 \mathrm{~kJ} / \mathrm{mol}$ reported by Lee et al. 69 Our CBS value may be considered the most accurate to date, and all the discussed results suggest that the experimental $E_{0}$ value of $12.3 \pm 1.4 \mathrm{~kJ} / \mathrm{mol}^{72}$ is slightly too large.

\subsection{Non-relativistic equilibrium geometry values}

The dependence of the computed shielding constants on the treatment of electron correlation effects and the basis set is shown in Table 3. For each approximation to the wavefunction we estimate the complete basis set (CBS) value of the correction using the best available results. Considering the convergence of the results with both basis set and the values of $\Delta \mathrm{T}$, we can estimate the error bars of the final equilibrium geometry values to be approximately \pm 4 ppm for $\sigma(\mathrm{N})$ and $\pm 2 \mathrm{ppm}$ for $\sigma(\mathrm{F})$.

The spin-spin coupling constants of FNNF have been previously computed (with a variety of basis sets) at the CCSD level in ref 19; here we use as the benchmark the CCSD/AV5Z-Juc results. At the CC3 level, the largest basis set that can be practically used for computational reasons is AVTZ-Juc (for the SD terms the smaller AVDZ-J basis set had to be used). The comparison of CC3 results with the corresponding CCSD values obtained for the same geometry is presented in Table 4. The difference between CCSD and CC3, $\Delta$ CC3, which is in many cases significant, is very large when individual components of the coupling constants are considered. In particular for ${ }^{3} J(\mathrm{FF})$ in both isomers the FC contribution to $\Delta \mathrm{CC} 3$ is on the order of $20 \mathrm{~Hz}$, whereas for the PSO term it is similar in magnitude but negative - thus the corrections to the CCSD values of the total ${ }^{3} J(\mathrm{FF})$ couplings are smaller.

\subsection{Vibrational corrections}

In the analysis of the vibrational corrections to the shielding constants we have considered a number of approximations. As shown in Table 5 the results practically do not differ significantly; changing the basis set applied to describe the force field or the shielding surface does not essentially affect the final results. For both nuclei in both isomers the vibrational corrections $\Delta \mathrm{Vib}(0 \mathrm{~K})$ are quite large, and significantly larger than the residual errors of the val- 
ues computed for the equilibrium geometries. The $\Delta$ Temp values (showing the additional corrections for $300 \mathrm{~K}$ ), practically negligible for nitrogen, are also significant for fluorine shielding.

We conclude that anharmonic effects are significant for N-F bonds, which is consistent with our previous study of $\mathrm{NF}_{3}, 15$ where vibrational corrections were significantly greater in $\mathrm{NF}_{3}$ in comparison with $\mathrm{PF}_{3}$ and $\mathrm{AsF}_{3}$.

It is obvious that vibrational corrections have to be included when comparing the computed shielding constants with experimental data, both for a genuine comparison of structures, and due to the magnitude of the effect.

Vibrational corrections to the coupling constants at $300 \mathrm{~K}$ are collected in Table 6 For both isomers, the corrections are large for ${ }^{1} J(\mathrm{NF})$ and ${ }^{3} J(\mathrm{FF})$ couplings, and much smaller for ${ }^{1} J(\mathrm{NN})$ and ${ }^{2} J(\mathrm{NF})$. However, considering the size of the coupling constants, they are in percentage terms not really larger than the corrections to the other couplings, 3-4\%. Compared to the CC3 corrections to the couplings, the vibrational corrections to the ${ }^{1} J(\mathrm{NF})$ and ${ }^{3} J(\mathrm{FF})$ couplings in cis-FNNF and the ${ }^{1} J(\mathrm{NN})$ and ${ }^{1} J(\mathrm{NF})$ couplings in trans-FNNF are actually larger. This shows again that inclusion of vibrational corrections can be as important as the inclusion of coupled-cluster triples corrections, as observed previously for FCCF. 12

The results, obtained using either the AVQZ or the AVTZ-J basis set for the calculation of the coupling constant derivatives, are very similar, with the exception of the FC contribution to ${ }^{3} J(F F)$ in the cis isomer where the difference in sign leads to a significant difference in the total vibrational correction. A smaller, but also noticeable difference is also observed for the FC contribution to the ${ }^{3} J(\mathrm{FF})$ coupling in the trans isomer. To estimate the total coupling constants we have utilized in the following the a priori more reliable AVTZ-J basis set results for coupling derivatives, although we note here that using the AVQZ-calculated value for ${ }^{3} J(\mathrm{FF})$ in the cis isomer would lead to an improved agreement with experiment.

\subsection{Relativistic corrections}

To estimate the role of the relativistic effects we compare the results obtained at the DFT four-component level with their nonrelativistic counterparts. In this analysis of the relativistic corrections we used Dyall's basis sets (see refs 73/74 and references therein). The results for the shielding and spin-spin coupling constants, obtained at the CCSD(T)/aug-cc-pCVQZ geometry, are shown in Table 7 and Table 8, respectively. While the calculated shielding and spin-spin coupling constants strongly depend on the method and basis set, the relativistic corrections are similar for HF and the various DFT functionals considered.

Relativistic and non-relativistic values of the shielding constants have been obtained in the Dirac-Coulomb (DC) and LévyLeblond (LL) approach, respectively, using the ReSpect program. As shown in Table 7 the relativistic corrections are not negligible; we shall use the CBS values obtained with the KT2 functional to estimate the final total shielding constants. The convergence with the extension of the basis set is satisfying, and the dependence on the functional is very weak.
Relativistic corrections to the spin-spin coupling constants (also obtained with the ReSpect program) are small in magnitude, but more dependent on the DFT functional chosen within the DiracKohn-Sham approach than the shieldings. In the following we have utilized the PBE0-50 (PBE0 functional with the HartreeFock exchange part increased from $25 \%$ to $50 \%$ ) corrections, because this functional gives the best approximation to the spin-spin coupling constants. For example, the 4-component relativistic HF, BP86, PBE and PBE0-50 values of ${ }^{3} J(F F)$ in the cis isomer are $-330,-211,-200$ and $-165 \mathrm{~Hz}$, respectively, whereas the experimental value is $-146 \mathrm{~Hz}$. Furthermore for both isomers the PBE050 values of the ${ }^{3} J$ (FF) constants lie within $12 \%$ of the coupledcluster results.

\subsection{Comparison with experiment and previous calculations}

To compare the calculated shielding constants with experimental data, we have determined experimental shielding constants by combining literature values of $\sigma_{r e f}\left(\mathrm{~F}\right.$ in $\left.\mathrm{CFCl}_{3}\right)$ and $\sigma_{\text {ref }}\left(\mathrm{N}\right.$ in $\left.\mathrm{NF}_{3}\right)$ and the experimental values of the chemical shifts. As shown in Table 9 the agreement of the nitrogen shielding constants with experiment is excellent for both isomers, with differences of less than $2 \mathrm{ppm}$. This suggests that the much larger differences in the fluorine shielding constants do not reflect solely the role of the approximations made in the computational approach. In particular, the discrepancy between calculated shielding constants and the experimental values may largely be due to solvent effects; the observed ${ }^{19} \mathrm{~F}$ gas-to-liquid shifts are often on the order of $10 \mathrm{ppm} .75 / 76$ The deviation between calculated (gas phase) and experimental (solvated) ${ }^{19} \mathrm{~F}$ shielding of the cis and trans isomers is 15.7 and $11.1 \mathrm{ppm}$, respectively. Adding such a solvent correction of $-10 \mathrm{ppm}$ to the computed isolated molecule (gas phase) values would bring the results for both isomers much closer to the experimental data.

Comparison of the calculated spin-spin coupling constants with the experimental values in Table 10 shows very good agreement for the trans isomer with differences of less than $1 \mathrm{~Hz}(1 \%$ or less) for the one-bond and two-bond couplings and $4 \%$ or $13 \mathrm{~Hz}$ for the three-bond fluorine-fluorine coupling. For the cis isomer the differences are consistently somewhat larger: between 2 and $4 \mathrm{~Hz}$ for the one- and two-bond couplings and with $19 \mathrm{~Hz}$ again larger for the three-bond coupling. However, the latter is percentwise not larger than for the two-bond coupling in the cis isomer.

Our coupled-cluster results permit an estimate of the quality of the old MCSCF values; in this comparison we shall focus on those obtained with the largest active space, called RAS-E. 18 For the shielding constants (see Table 9) the agreement of the present coupled-cluster results with the experimental data is clearly better (for fluorine shielding the CAS-5252 values obtained with a larger basis set are much closer to both our present and experimental results). On the other hand, as shown in Table 10, the RAS-E spin-spin coupling constants are surprisingly close to the present results, in particular for the cis isomer. Only for ${ }^{1} J(\mathrm{NF})$ in the cis isomer and for ${ }^{1} J(\mathrm{NN})$ and ${ }^{3} J(\mathrm{FF})$ in the trans isomer are our new results undeniably better than the MCSCF values. 


\section{Conclusions}

Extensive coupled-cluster calculations have enabled NMR shielding and spin-spin coupling constants to be determined to nearquantitative accuracy that is competitive with experiment for both cis- and trans-FNNF.

For ${ }^{15} \mathrm{~N}$ shielding constants, the agreement between theory and experiment is excellent, being within $2 \mathrm{ppm}$. For the ${ }^{19} \mathrm{~F}$ shielding constants there remains a discrepancy between experimental and calculated values, which may be rationalized by sizeable solvent effects on fluorine shielding. It is concluded that the present calculated gas-phase shielding constants provide the most accurate values to date, and that the available experimental chemical shift does not accurately represent a single-molecule gas phase value. Experimental gas-phase shielding values would be needed for confirmation. Vibrational effects are large for ${ }^{19} \mathrm{~F}$ shielding constants, and must be considered if N-F bonds are present in a molecule.

For spin-spin coupling constants, extensive CC3 calculations have been carried out. The difference between CC3 and CCSD results, $\Delta \mathrm{CC} 3$, is particularly significant for ${ }^{1} J(\mathrm{NF})$ and ${ }^{2} J(\mathrm{NF})$ coupling constants. For the cis isomer, including $\Delta \mathrm{CC} 3$ corrections improves the agreement with experimental data, while for the trans isomer it brings both calculated values into excellent agreement with experiment.

For ${ }^{1} J(\mathrm{NN})$ in the cis isomer, agreement of calculated constant is improved noticeably when $\Delta \mathrm{CC} 3$ is included; for the trans isomer the present results confirm that ${ }^{1} J(\mathrm{NN})$ is negative (coupling constants were not directly observed in $[\mathrm{AX}]_{2}$ NMR experiment, they were extracted from linear combinations detectable in the spectra and various sign options have been discussed in ref 78).

For ${ }^{3} J(\mathrm{FF})$ including the $\Delta \mathrm{CC} 3$ correction improves the agreement with the experimental value for the trans isomer, but deteriorates the agreement for the trans isomer. It appears, however, that for a reliable comparison with experiment, gas-phase values of ${ }^{3} J(\mathrm{FF})$ may be needed for both isomers since in solution the geometry of FNNF, and thus the F-F distance as well as vibrational effects, may be significantly affected by intermolecular forces.

For some of the couplings the vibrational corrections were found to be as large as the $\Delta \mathrm{CC} 3$ corrections showing again that a balanced description of electron correlation and vibrational correction contributions is necessary for achieving highly accurate values.

\section{Conflicts of interest}

There are no conflicts to declare.

\section{Acknowledgements}

The authors acknowledge generous allocations of computing resources from NCI, La Trobe University, Intersect, and the Department of Chemistry, University of Copenhagen.

\section{Notes and references}

1 Calculation of NMR and EPR parameters. Theory and applications, ed. M. Kaupp, M. Bühl and V. G. Malkin, Wiley-VCH, Weinheim, 2004.
2 T. Helgaker, S. Coriani, P. Jørgensen, K. Kristensen, J. Olsen and K. Ruud, Chem. Rev., 2012, 112, 543-631.

3 High Resolution NMR Spectroscopy: Understanding Molecules and their Electronic Structures, ed. R. H. Contreras, Elsevier, Amsterdam, 2013.

4 J. Dietschreit, A. Wagner, T.-A. Le, P. Klein, H. Schindelin, T. Opatz, B. Engels, U. Hellmich and C. Ochsenfeld, Angew. Chem. Int. Ed., 2020, 59, 12669-12673.

5 V. Barone, P. F. Provasi, J. E. Peralta, J. P. Snyder, S. P. A. Sauer and R. H. Contreras, J. Phys. Chem. A, 2003, 107, 4748-4754.

6 P. F. Provasi, G. A. Aucar and S. P. A. Sauer, J. Phys. Chem. A, 2004, 108, 5393-5398.

7 M. Sanchez, P. F. Provasi, G. A. Aucar and S. P. A. Sauer, Adv. Quantum Chem., 2005, 48, 161-183.

8 P. F. Provasi and S. P. A. Sauer, J. Chem. Theory Comput., 2006, 2, 1019-1027.

9 M. E. Harding, M. Lenhart, A. A. Auer and J. Gauss, J. Chem. Phys., 2008, 128, 244111.

10 H. Kjær, S. P. A. Sauer and J. Kongsted, J. Chem. Phys., 2010, 133, 144106.

11 P. F. Provasi, M. C. Caputo, S. P. A. Sauer, I. Alkorta and J. Elguero, Comp. Theor. Chem., 2012, 998, 98-105.

12 R. Faber and S. P. A. Sauer, Phys. Chem. Chem. Phys., 2012, 14, 16440-16447.

13 M. C. Caputo, I. Alkorta, P. F. Provasi and S. P. A. Sauer, Mol. Phys., 2018, 116, 2396-2405.

14 R. Faber, S. P. A. Sauer and J. Gauss, J. Chem. Theory Comp., 2017, 13, 696-709.

15 T. E. Field-Theodore, M. Olejniczak, M. Jaszuński and D. J. D. Wilson, Phys. Chem. Chem. Phys., 2018, 20, 23025-23033.

16 A. K. Schnack-Petersen, P. A. B. Haase, R. Faber, P. F. Provasi and S. P. A. Sauer, J. Comp. Chem., 2018, 39, 2647-2666.

17 A. Rizzo, C. Cappelli, B. Jansik, D. Jonsson, P. Sałek, S. Coriani, D. J. D. Wilson, T. Helgaker and H. Ågren, J. Chem. Phys., 2005, 122, 234314.

18 M. Jaszuński, T. Helgaker and K. Ruud, Magn. Reson. Chem., 1996, 34, 646-649.

19 R. Faber and S. P. A. Sauer, Theor. Chem. Acc., 2018, 137, article 35.

20 F. London, J. Phys. Radium, 1937, 8, 397-409.

21 J. Gauss and J. F. Stanton, J. Chem. Phys., 1995, 102, 251253.

22 J. Gauss and J. F. Stanton, J. Chem. Phys., 1996, 104, 2574 2583.

23 J. Gauss, J. Chem. Phys., 2002, 116, 4773-4776.

24 M. Kállay and J. Gauss, J. Chem. Phys., 2004, 120, 68416848.

25 S. Coriani, C. Hättig, P. Jørgensen and T. Helgaker, J. Chem. Phys., 2000, 113, 3561-3572.

26 R. Ditchfield, J. Chem. Phys., 1972, 56, 5688-5691.

27 R. Ditchfield, Mol. Phys., 1974, 27, 789-807.

28 K. Wolinski, J. F. Hinton and P. Pulay, J. Am. Chem. Soc., 1990, 112, 8251-8260. 
29 D. Sundholm, J. Gauss and A. Schäfer, J. Chem. Phys., 1996, 105, 11051-11059.

30 A. A. Auer, J. Gauss and J. F. Stanton, J. Chem. Phys., 2003, 118, 10407-10417.

31 E. Prochnow and A. A. Auer, J. Chem. Phys., 2010, 132, 064109.

32 R. Faber and S. P. A. Sauer, AIP Conf. Proc., 2015, 1702, 90035.

33 R. Faber, A. Buczek, T. Kupka and S. P. A. Sauer, Mol. Phys., 2017, 115, 144-160.

34 R. Faber, J. Kaminsky and S. P. A. Sauer. Rovibrational and Temperature Effects in Theoretical Studies of NMR Parameters, in Gas Phase NMR , ed. K. Jackowski and M. Jaszuński, The Royal Society of Chemistry, 2016, ch. 7, pp. 218-266.

35 J. Autschbach. Relativistic Effects on NMR Parameters, in High Resolution NMR Spectroscopy: Understanding Molecules and their Electronic Structures, ed. R. H. Contreras, Elsevier, 2013, vol. 3, ch. 4, pp. 69-117.

36 M. Repisky, S. Komorovsky, R. Bast and K. Ruud. Relativistic Calculations of Nuclear Magnetic Resonance Parameters, in Gas Phase NMR, ed. K. Jackowski and M. Jaszuński, The Royal Society of Chemistry, 2016, ch. 8, pp. 267-303.

37 A. M. Teale, O. B. Lutnæs, T. Helgaker, D. J. Tozer and J. Gauss, J. Chem. Phys., 2013, 138, 024111.

38 L. Armangue, M. Sola and M. Swart, Mol. Phys., 2013, 111, 1332-1344.

39 S. R. Jensen, T. Flå, D. Jonsson, R. S. Monstad, K. Ruud and L. Frediani, Phys. Chem. Chem. Phys., 2016, 18, 2114521161.

40 M. Sun, I. Y. Zhang, A. Wu and X. Xu, J. Chem. Phys., 2013, 138, 124113.

41 P. J. P. de Oliveira and F. E. Jorge, J. Phys. B: At. Mol. Phys., 2008, 41, 145101.

42 T. Kupka, M. Stachów, M. Nieradka, J. Kaminsky, T. Pluta and S. P. A. Sauer, Magn. Reson. Chem., 2011, 49, 231-236.

43 T. Kupka, M. Stachów, J. Kaminsky and S. P. A. Sauer, Magn. Reson. Chem., 2013, 51, 482-489.

44 T. Kupka, M. Nieradka, M. Stachów, T. Pluta, P. Nowak, H. Kjær, J. Kongsted and J. Kaminsky, J. Phys. Chem. A, 2012, 116, 3728-3738.

45 T. Kupka and B. Ruscic and R. E. Botto, J. Phys. Chem. A, 2002, 106, 10396-10407.

46 T. Kupka, Magn. Reson. Chem., 2008, 46, 851-858.

47 J. Geertsen and J. Oddershede, Chem. Phys., 1984, 90, 301311.

48 T. Helgaker, M. Jaszuński and K. Ruud, Chem. Rev., 1999, 99, 293-352.

49 G. E. Scuseria, J. Geertsen and J. Oddershede, J. Chem. Phys., 1989, 90, 2338-2343.

50 P. F. Provasi, G. A. Aucar and S. P. A. Sauer, J. Chem. Phys., 2001, 115, 1324-1334.

51 O. B. Lutnæs, T. Helgaker and M. Jaszuński, Mol. Phys., 2010, 108, 2579-2590.

52 A. A. Auer and J. Gauss, Chem. Phys., 2009, 356, 7-13.
53 A. A. Auer and J. Gauss, J. Chem. Phys., 2001, 115, 16191622.

54 CFOUR, Coupled-Cluster techniques for Computational Chemistry, a quantum-chemical program package, J. F. Stanton and J. Gauss and L. Cheng and M. E. Harding and D. A. Matthews and P. G. Szalay, with contributions from A.A. Auer, R.J. Bartlett, U. Benedikt, C. Berger, D.E. Bernholdt, Y.J. Bomble, O. Christiansen, F. Engel, R. Faber, M. Heckert, O. Heun, M. Hilgenberg, C. Huber, T.-C. Jagau, D. Jonsson, J. Jusélius, T. Kirsch, K. Klein, W.J. Lauderdale, F. Lipparini, T. Metzroth, L.A. Mück, D.P. O’Neill, D.R. Price, E. Prochnow, C. Puzzarini, K. Ruud, F. Schiffmann, W. Schwalbach, C. Simmons, S. Stopkowicz, A. Tajti, J. Vázquez, F. Wang, J.D. Watts and the integral packages MOLECULE (J. Almlöf and P.R. Taylor), PROPS (P.R. Taylor), ABACUS (T. Helgaker, H.J. Aa. Jensen, P. Jørgensen, and J. Olsen), and ECP routines by A. V. Mitin and C. van Wüllen. For the current version, see http://www.cfour.de.

55 MRCC, a quantum chemical program suite written by M. Kállay, P. R. Nagy, D. Mester, Z. Rolik, G. Samu, J. Csontos, J. Csóka, P. B. Szabó, L. Gyevi-Nagy, B. Hégely, I. Ladjánszki, L. Szegedy, B. Ladóczki, K. Petrov, M. Farkas, P. D. Mezei, and Á. Ganyecz. See www.mrcc.hu.

56 M. Kállay, P. R. Nagy, D. Mester, Z. Rolik, G. Samu, J. Csontos, J. Csóka, P. B. Szabó, L. Gyevi-Nagy, B. Hégely, I. Ladjánszki, L. Szegedy, B. Ladóczki, K. Petrov, M. Farkas, P. D. Mezei and Á. Ganyecz, J. Chem. Phys., 2020, 152, 074107.

57 ReSpect, version 5.1.0 (2018); Relativistic Spectroscopy DFT program of authors Repisky, M.; Komorovsky, S.; Malkin, V. G.; Malkina, O. L.; Kaupp, M.; Ruud, K., with contributions from Bast, R.; Ekström, U.; Kadek, M.; Knecht, S.; Konecny, L.; Malkin, E.; Malkin-Ondik, I. (see www.respectprogram.org).

58 T. H. Dunning Jr., J. Chem. Phys., 1989, 90, 1007-1023.

59 R. A. Kendall, T. H. Dunning Jr. and R. J. Harrison, J. Chem. Phys., 1992, 96, 6796-6806.

60 D. E. Woon and T. H. Dunning Jr., J. Chem. Phys., 1993, 98, 1358-1371.

61 D. E. Woon and T. H. Dunning Jr., J. Chem. Phys., 1995, 103, 4572-4585.

62 A. K. Wilson, D. E. Woon, K. A. Peterson and T. H. Dunning Jr., J. Chem. Phys., 1999, 110, 7667-7676.

63 K. A. Peterson and T. H. Dunning Jr., J. Chem. Phys., 2002, 117, 10548-10560.

64 J. G. Hill, S. Mazumder and K. A. Peterson, J. Chem. Phys., 2010, 132, 054108.

65 U. Benedict, A. A. Auer and F. Jensen, J. Chem. Phys., 2008, 129, 064111.

66 T. A. Ruden and K. Ruud, Ro-Vibrational Corrections to NMR Parameters, in Calculation of NMR and EPR parameters. Theory and applications, eds. M. Kaupp, M. Bühl and V. G. Malkin, Weinheim, 2004, pp. 153-173.

67 R. L. Kuczkowski and E. B. Wilson, J. Chem. Phys., 1963, 39, 1030-1034.

68 R. K. Bohn and S. H. Bauer, Inorg. Chem., 1967, 6, 309-312.

69 T. J. Lee, J. E. Rice, G. E. Scuseria and H. F. Schaefer III, 
Theor. Chim. Acta, 1989, 75, 81-98.

70 K. O. Christe, D. A. Dixon, D. J. Grant, R. Haiges, F. S. Tham, A. Vij, V. Vij, T.-H. Wang and W. W. Wilson, Inorg. Chem., 2010, 49, 6823-6833.

71 T. J. Lee and P. R. Taylor, Int. J. Quantum Chem., 1989, 36, 199-207.

72 N. C. Craig, L. G. Piper and V. L. Wheeler, J. Phys. Chem., 1971, 75, 1453-1460.

73 K. G. Dyall, Unpublished Basis Sets for $2 p$ - 3p Elements, Available from the Dirac web site, http://dirac.chem.sdu.dk.
74 K. G. Dyall, Theor. Chem. Acc., 2016, 135, 128-136.

75 C. J. Jameson, A. K. Jameson and P. M. Burrell, J. Chem. Phys., 1980, 73, 6013-6020.

76 C. J. Jameson, A. K. Jameson and J. Honarbakhsh, J. Chem. Phys., 1984, 81, 5266-5267.

77 J. H. Noggle, J. D. Baldeschwieler and C. B. Colburn, J. Chem. Phys., 1962, 37, 182-189.

78 F. Aubke, G. Hägele and H. Willner, Magn. Reson. Chem., 1995, 33, 817-822. 
Table 1 Experimental and calculated geometries of cis and trans-FNNF. Bond distances in units of $\AA$, angles in degrees

\begin{tabular}{|c|c|c|c|c|c|c|}
\hline & \multicolumn{2}{|c|}{$\overline{\text { Experiment }}$} & \multirow{2}{*}{$\begin{array}{l}\mathrm{MP2}^{c} \\
\text { TZ2Pf }\end{array}$} & \multirow{2}{*}{$\begin{array}{c}\operatorname{CCSD}(\mathrm{T})^{d} \\
\text { AVTZ }\end{array}$} & \multirow{2}{*}{$\begin{array}{c}\mathrm{CCSD}(\mathrm{T})^{e} \\
\text { ACVQZ }\end{array}$} & \multirow{2}{*}{$\begin{array}{c}\mathrm{CCSD}(\mathrm{T})^{f} \\
\text { ACV5Z }\end{array}$} \\
\hline & $\operatorname{MW}(1963)^{a}$ & $\mathrm{ED}(1967)^{b}$ & & & & \\
\hline \multicolumn{7}{|l|}{ cis } \\
\hline $\mathrm{r}(\mathrm{NN})$ & $1.214 \pm 0.005$ & $1.213 \pm 0.012$ & 1.224 & 1.225 & 1.219 & 1.218 \\
\hline$r(N F)$ & $1.384 \pm 0.010$ & $1.410 \pm 0.009$ & 1.386 & 1.387 & 1.379 & 1.377 \\
\hline$\angle(N N F)$ & $114.5 \pm 0.5$ & $114.4 \pm 1.0$ & 114.4 & 114.2 & 114.28 & 114.28 \\
\hline \multicolumn{7}{|l|}{ trans } \\
\hline $\mathrm{r}(\mathrm{NN})$ & & $1.230 \pm 0.010$ & 1.233 & 1.232 & 1.226 & 1.225 \\
\hline $\mathrm{r}(\mathrm{NF})$ & & $1.396 \pm 0.008$ & 1.384 & 1.386 & 1.377 & 1.375 \\
\hline$\angle$ (NNF) & & $105.5 \pm 0.7$ & 104.6 & 104.7 & 105.04 & 105.08 \\
\hline
\end{tabular}

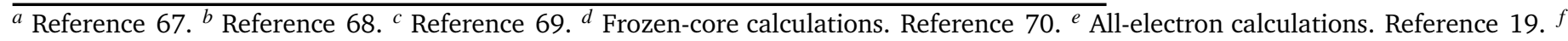
This work. All-electron calculations.

Table 2 Calculated trans-cis isomerization energy of FNNF. Units of $\mathrm{kJ} / \mathrm{mol}$

\begin{tabular}{lcccrrr}
\hline \hline \multirow{2}{*}{ Contr. $^{a}$} & ACVDZ & ACVTZ & ACVQZ & ACV5Z & ACV6Z & ACV7Z \\
\hline \hline SCF & -5.971 & -6.086 & -6.218 & -6.228 & -6.228 & -6.228 \\
$\Delta$ SD & 2.700 & 3.445 & 3.583 & 3.566 & -6.228 \\
$\Delta(\mathrm{T})$ & 9.348 & 8.287 & 8.334 & 8.411 & 3.549 \\
$\Delta \mathrm{T}$ & -0.024 & 0.026 & 0.029 & & 8.491 \\
$\Delta$ Q & 0.352 & & & & 0.032 \\
$\Delta$ Rel. $^{b}$ & & 0.081 & 0.079 & & 0.352 \\
$\Delta$ ZPVE $^{c}$ & & & & & 0.077 \\
Sum & & & & & -0.633 \\
\hline \hline
\end{tabular}

a Contribution: SCF is the Hartree-Fock value; $\Delta$ SD is CCSD - SCF correction; $\Delta$ (T) is CCSD(T) - CCSD correction; $\Delta$ T is CCSDT - CCSD(T) correction; $\Delta \mathrm{Q}$ is CCSDTQ - CCSDT correction. The $\Delta \mathrm{T}$ and $\Delta \mathrm{Q}$ contributions were calculated with the frozen-core approximation. $\Delta \mathrm{Q}$ uses a dzp basis set. ${ }^{b}$ PBE0 with dyall-ACVnZ $(\mathrm{n}=3,4)$ basis sets (see Table 7). ${ }^{c}$ Zero-point vibrational energy with all-electron CCSD(T)/ACVQZ. Experimental value is -0.473 $\mathrm{kJ} / \mathrm{mol}$.

Table 3 Calculated $\operatorname{CCSD}(\mathrm{T}) / \mathrm{ACVQZ}$ equilibrium geometry contributions to the $\mathrm{N}$ and $\mathrm{F}$ isotropic shielding in FNNF. Units of ppm

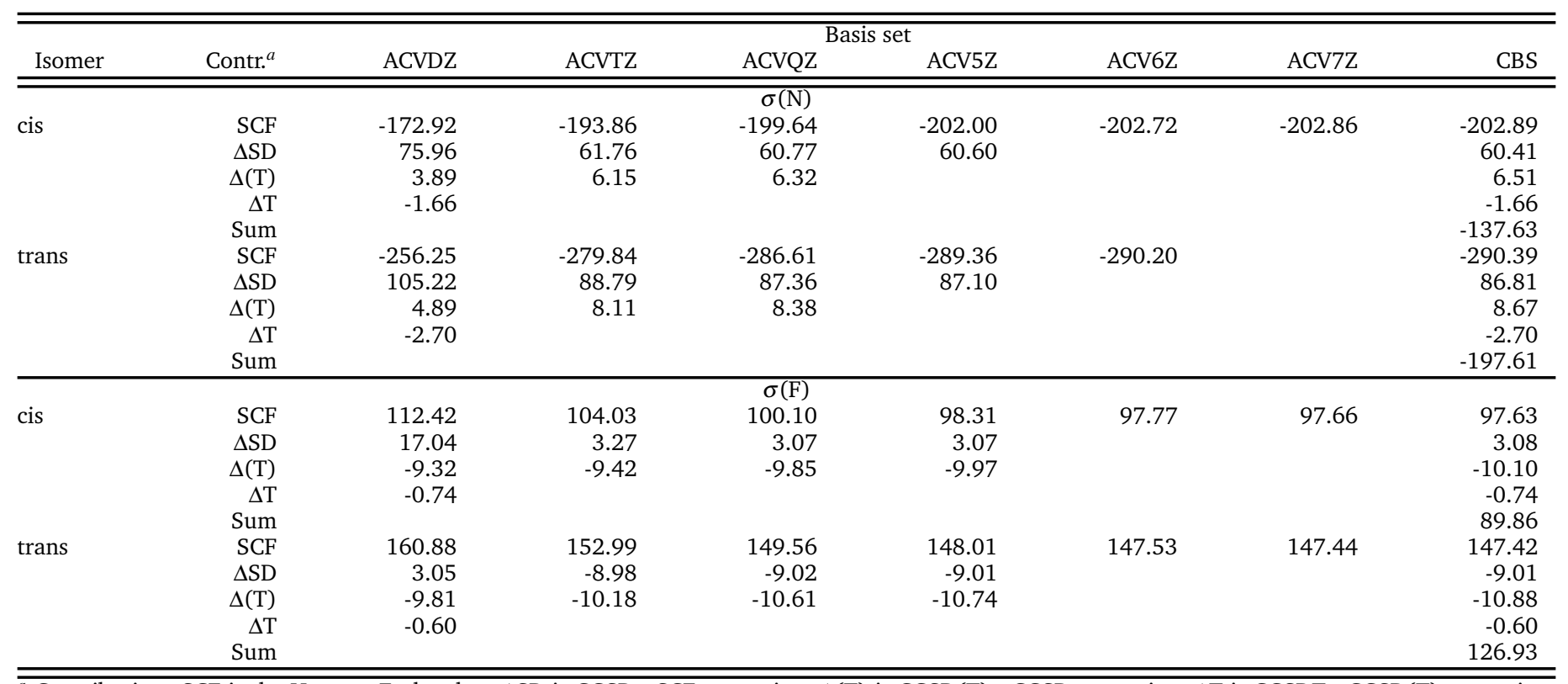

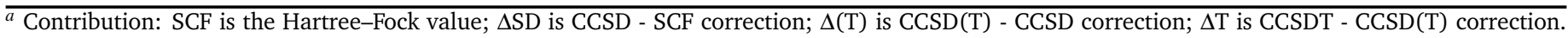
For $\Delta \mathrm{T}$ both CCSDT and $\operatorname{CCSD}(\mathrm{T})$ contributions were calculated with tz2p basis set. 
Table 4 Contributions to the $\operatorname{CSSD}(\mathrm{T}) / \mathrm{ACVQZ}$ equilibrium geometry values of the coupling constants in FNNF. Units of $\mathrm{Hz}$

\begin{tabular}{|c|c|c|c|c|c|c|c|c|}
\hline Isomer & Coupling & Method & Basis set & $\overline{\text { FC }}$ & $\overline{\mathrm{SD}^{b}}$ & $\overline{\mathrm{PSO}}$ & $\overline{\mathrm{DSO}}$ & total \\
\hline \multirow[t]{4}{*}{$\overline{\text { cis }}$} & ${ }^{1} J(\mathrm{NN})$ & $\begin{array}{l}\mathrm{CCSD}^{a} \\
\mathrm{CCSD}^{a} \\
\mathrm{CC} 3 \\
\Delta \mathrm{CC}^{c}\end{array}$ & $\begin{array}{l}\text { AV5Z-Juc } \\
\text { AVTZ-Juc } \\
\text { AVTZ-Juc } \\
\text { AVTZ-Juc }\end{array}$ & $\begin{array}{l}2.67 \\
2.71 \\
4.01 \\
1.30\end{array}$ & $\begin{array}{r}1.76 \\
1.69 \\
1.57 \\
-0.12\end{array}$ & $\begin{array}{r}-4.54 \\
-4.51 \\
-4.19 \\
0.32\end{array}$ & $\begin{array}{l}0.03 \\
0.03 \\
0.03 \\
0.00\end{array}$ & $\begin{array}{r}-0.08 \\
-0.05 \\
1.41 \\
1.49\end{array}$ \\
\hline & ${ }^{1} J(\mathrm{NF})$ & $\begin{array}{l}\operatorname{CCSD}^{a} \\
\operatorname{CCSD}^{a} \\
\text { CC3 } \\
\Delta \text { CC3 }^{c}\end{array}$ & $\begin{array}{l}\text { AV5Z-Juc } \\
\text { AVTZ-Juc } \\
\text { AVTZ-Juc } \\
\text { AVTZ-Juc }\end{array}$ & $\begin{array}{r}244.42 \\
243.56 \\
244.36 \\
0.80\end{array}$ & $\begin{array}{r}-19.64 \\
-18.73 \\
-17.87 \\
0.86\end{array}$ & $\begin{array}{r}-12.13 \\
-11.87 \\
-10.03 \\
1.84\end{array}$ & $\begin{array}{r}-0.33 \\
-0.33 \\
-0.33 \\
0.00\end{array}$ & $\begin{array}{r}212.32 \\
212.20 \\
216.13 \\
3.50\end{array}$ \\
\hline & ${ }^{2} J(\mathrm{NF})$ & $\begin{array}{l}\operatorname{CCSD}^{a} \\
\operatorname{CCSD}^{a} \\
\text { CC3 } \\
\Delta \text { CC3 }^{c}\end{array}$ & $\begin{array}{l}\text { AV5Z-Juc } \\
\text { AVTZ-Juc } \\
\text { AVTZ-Juc } \\
\text { AVTZ-Juc }\end{array}$ & $\begin{array}{r}-47.57 \\
-47.81 \\
-54.09 \\
-6.28\end{array}$ & $\begin{array}{l}-2.13 \\
-2.14 \\
-2.49 \\
-0.35\end{array}$ & $\begin{array}{r}34.98 \\
34.81 \\
35.51 \\
0.70\end{array}$ & $\begin{array}{l}0.17 \\
0.17 \\
0.17 \\
0.00\end{array}$ & $\begin{array}{r}-14.54 \\
-14.98 \\
-20.90 \\
-5.93\end{array}$ \\
\hline & ${ }^{3} J(\mathrm{FF})$ & $\begin{array}{l}\operatorname{CCSD}^{a} \\
\operatorname{CCSD}^{a} \\
\mathrm{CC} 3 \\
\Delta \mathrm{CC}^{c}\end{array}$ & $\begin{array}{l}\text { AV5Z-Juc } \\
\text { AVTZ-Juc } \\
\text { AVTZ-Juc } \\
\text { AVTZ-Juc }\end{array}$ & $\begin{array}{r}150.18 \\
152.75 \\
175.17 \\
22.42\end{array}$ & $\begin{array}{r}42.03 \\
42.13 \\
44.29 \\
2.16\end{array}$ & $\begin{array}{r}-319.73 \\
-320.12 \\
-341.41 \\
-21.29\end{array}$ & $\begin{array}{l}0.04 \\
0.04 \\
0.05 \\
0.01\end{array}$ & $\begin{array}{r}-127.48 \\
-125.39 \\
-121.90 \\
3.30\end{array}$ \\
\hline \multirow[t]{4}{*}{ trans } & ${ }^{1} J(\mathrm{NN})$ & $\begin{array}{l}\mathrm{CCSD}^{a} \\
\mathrm{CCSD}^{a} \\
\mathrm{CC} 3 \\
\Delta \mathrm{CC}^{c}\end{array}$ & $\begin{array}{l}\text { AV5Z-Juc } \\
\text { AVTZ-Juc } \\
\text { AVTZ-Juc } \\
\text { AVTZ-Juc }\end{array}$ & $\begin{array}{r}-10.33 \\
-10.27 \\
-10.67 \\
-0.40\end{array}$ & $\begin{array}{r}1.43 \\
1.39 \\
1.27 \\
-0.12\end{array}$ & $\begin{array}{r}-8.66 \\
-8.56 \\
-8.26 \\
0.30\end{array}$ & $\begin{array}{l}0.02 \\
0.02 \\
0.03 \\
0.01\end{array}$ & $\begin{array}{r}-17.53 \\
-17.41 \\
-17.64 \\
-0.22\end{array}$ \\
\hline & ${ }^{1} J(\mathrm{NF})$ & $\begin{array}{l}\operatorname{CCSD}^{a} \\
\operatorname{CCSD}^{a} \\
\mathrm{CC} 3 \\
\Delta \mathrm{CC}^{c}\end{array}$ & $\begin{array}{l}\text { AV5Z-Juc } \\
\text { AVTZ-Juc } \\
\text { AVTZ-Juc } \\
\text { AVTZ-Juc }\end{array}$ & $\begin{array}{r}220.41 \\
219.58 \\
219.41 \\
-0.17\end{array}$ & $\begin{array}{r}-21.06 \\
-20.15 \\
-19.56 \\
0.59\end{array}$ & $\begin{array}{r}-21.47 \\
-21.18 \\
-19.66 \\
1.52\end{array}$ & $\begin{array}{l}-0.30 \\
-0.30 \\
-0.31 \\
-0.01\end{array}$ & $\begin{array}{r}177.58 \\
177.54 \\
179.89 \\
1.94\end{array}$ \\
\hline & ${ }^{2} J(\mathrm{NF})$ & $\begin{array}{l}\operatorname{CCSD}^{a} \\
\operatorname{CCSD}^{a} \\
\mathrm{CC} 3 \\
\Delta \mathrm{CC}^{c}\end{array}$ & $\begin{array}{l}\text { AV5Z-Juc } \\
\text { AVTZ-Juc } \\
\text { AVTZ-Juc } \\
\text { AVTZ-Juc }\end{array}$ & $\begin{array}{r}-62.10 \\
-62.11 \\
-65.02 \\
-2.91\end{array}$ & $\begin{array}{l}-6.29 \\
-6.21 \\
-6.38 \\
-0.17\end{array}$ & $\begin{array}{r}9.67 \\
9.63 \\
9.61 \\
-0.02\end{array}$ & $\begin{array}{l}0.06 \\
0.06 \\
0.06 \\
0.00\end{array}$ & $\begin{array}{c}-58.66 \\
-58.68 \\
-61.74 \\
-3.11\end{array}$ \\
\hline & ${ }^{3} J(\mathrm{FF})$ & $\begin{array}{l}\operatorname{CCSD}^{a} \\
\operatorname{CCSD}^{a} \\
\mathrm{CC} 3 \\
\Delta \mathrm{CC}^{c}\end{array}$ & $\begin{array}{l}\text { AV5Z-Juc } \\
\text { AVTZ-Juc } \\
\text { AVTZ-Juc } \\
\text { AVTZ-Juc }\end{array}$ & $\begin{array}{l}21.26 \\
23.61 \\
40.41 \\
16.80\end{array}$ & $\begin{array}{r}52.05 \\
49.98 \\
49.94 \\
-0.04\end{array}$ & $\begin{array}{r}-372.86 \\
-373.13 \\
-397.63 \\
-24.50\end{array}$ & $\begin{array}{r}-2.29 \\
-2.28 \\
-2.28 \\
0.00\end{array}$ & $\begin{array}{r}-301.83 \\
-300.06 \\
-309.56 \\
-7.7\end{array}$ \\
\hline
\end{tabular}

${ }^{a}$ AV5Z-Juc results from ref [19, primary but unpublished data from ref $19 \mid$ for the smaller basis sets.

${ }^{b}$ For comparison of the SD terms with CC3 instead of AVTZ-Juc results we report here AVDZ-J values (unpublished data from ref 19).

${ }^{c}$ Calculated as the difference between the CC3 and CCSD results obtained with the same basis set, i.e. AVTZ-Juc for the FC, PSO and DSO terms and AVDZ-J for the SD term.

Table 5 Calculated vibrational corrections to the isotropic shieldings in FNNF. Units of ppm

\begin{tabular}{|c|c|c|c|c|c|c|c|}
\hline \multirow{2}{*}{$\overline{\text { Isomer }}$} & \multirow{2}{*}{$\overline{\text { Method }}$} & \multicolumn{2}{|c|}{$\overline{\overline{\text { Basis set }}}{ }^{a}$} & \multicolumn{2}{|c|}{$\overline{\sigma \sigma(\mathrm{N})}$} & \multicolumn{2}{|c|}{$\overline{\sigma(\mathrm{F})}$} \\
\hline & & Force field & NMR & $\Delta \operatorname{Vib}(0 \mathrm{~K})$ & $\Delta \mathrm{Temp}$ & $\Delta \operatorname{Vib}(0 \mathrm{~K})$ & $\Delta$ Temp \\
\hline \multirow[t]{3}{*}{$\overline{\text { cis }}$} & $\overline{C C S D(T)}$ & $\overline{\text { tz2p }}$ & $\overline{\text { tz2p }}$ & $\overline{-5.10}$ & -0.12 & -17.66 & $\overline{-1.83}$ \\
\hline & $\operatorname{CCSD}(\mathrm{T})$ & tz2p & gz2p & -5.20 & -0.12 & -17.59 & -1.80 \\
\hline & $\operatorname{CCSD}(\mathrm{T})$ & ACVTZ & ACVTZ & -5.16 & -0.12 & -17.46 & -1.81 \\
\hline \multirow[t]{3}{*}{ trans } & $\operatorname{ccsD}(\mathrm{T})$ & $\mathrm{tz} 2 \mathrm{p}$ & $\mathrm{tz} 2 \mathrm{p}$ & -5.67 & -0.59 & -15.70 & -1.90 \\
\hline & $\operatorname{CCSD}(\mathrm{T})$ & $\mathrm{tz} 2 \mathrm{p}$ & qz2p & -5.90 & -0.61 & -16.05 & -1.95 \\
\hline & $\operatorname{CCSD}(\mathrm{T})$ & ACVTZ & ACVTZ & -6.07 & -0.61 & -16.58 & -2.00 \\
\hline
\end{tabular}

${ }^{a}$ The force field basis set is the basis set employed in the cubic force field calculation; the NMR basis set was used for the calculation of NMR shielding derivatives for the vibrational averaging calculation. 
Table 6 Calculated CCSD vibrational corrections at $300 \mathrm{~K}$ to the coupling constants in FNNF. Units of $\mathrm{Hz}$

\begin{tabular}{|c|c|c|c|c|c|c|c|c|}
\hline \multirow[t]{2}{*}{$\overline{\overline{\text { Isomer }}}$} & \multirow[t]{2}{*}{$\overline{\overline{\text { Coupling }}}$} & \multicolumn{2}{|c|}{$\overline{\text { Basis set }}{ }^{a}$} & \multicolumn{5}{|c|}{$\bar{\Delta} \Delta \mathrm{Vib}(300 \mathrm{~K})$} \\
\hline & & Force field & NMR & FC & SD & PSO & DSO & total \\
\hline \multirow[t]{8}{*}{$\overline{\text { cis }}$} & $\overline{{ }^{1} J(\mathrm{NN})}$ & $\overline{\overline{\mathrm{AVQZ}^{b}}}$ & $\overline{\overline{\text { AVQZ }^{b}}}$ & 0.61 & 0.06 & 0.03 & 0.00 & 0.70 \\
\hline & & AVQZ & AVTZ-J ${ }^{c}$ & 0.57 & 0.06 & 0.03 & 0.00 & 0.65 \\
\hline & ${ }^{1} J(\mathrm{NF})$ & $\mathrm{AVQZ}^{b}$ & $\mathrm{AVQZ}^{b}$ & -6.48 & -0.64 & -0.79 & 0.01 & -7.90 \\
\hline & & AVQZ & AVTZ-J ${ }^{c}$ & -5.65 & -0.65 & -0.79 & 0.01 & -7.09 \\
\hline & ${ }^{2} J(\mathrm{NF})$ & $\mathrm{AVQZ}^{b}$ & $\mathrm{AVQZ}^{b}$ & -0.61 & -0.04 & 0.30 & 0.00 & -0.35 \\
\hline & & AVQZ & AVTZ-J ${ }^{c}$ & -1.08 & -0.04 & 0.30 & 0.00 & -0.82 \\
\hline & ${ }^{3} J(\mathrm{FF})$ & $\mathrm{AVQZ}^{b}$ & $\mathrm{AVQZ}^{b}$ & -3.57 & 4.18 & -11.79 & -0.01 & -11.19 \\
\hline & & AVQZ & AVTZ-J ${ }^{c}$ & 2.87 & 4.28 & -12.02 & -0.01 & -4.88 \\
\hline \multirow[t]{8}{*}{ trans } & ${ }^{1} J(\mathrm{NN})$ & $\mathrm{AVQZ}^{b}$ & $\mathrm{AVQZ}^{b}$ & -0.28 & 0.05 & -0.07 & 0.00 & -0.29 \\
\hline & & AVQZ & AVTZ-J ${ }^{c}$ & -0.28 & 0.06 & -0.07 & 0.00 & -0.29 \\
\hline & ${ }^{1} J(\mathrm{NF})$ & $\mathrm{AVQZ}^{b}$ & $\mathrm{AVQZ}^{b}$ & -5.77 & -0.65 & -0.89 & 0.01 & -7.31 \\
\hline & & AVQZ & AVTZ-J ${ }^{c}$ & -5.11 & -0.67 & -0.90 & 0.01 & -6.67 \\
\hline & ${ }^{2} J(\mathrm{NF})$ & $\mathrm{AVQZ}^{b}$ & AVQZ $^{b}$ & 0.35 & -0.01 & -0.01 & 0.00 & 0.33 \\
\hline & & AVQZ & AVTZ-J ${ }^{c}$ & 0.15 & -0.02 & -0.01 & 0.00 & 0.12 \\
\hline & ${ }^{3} J(\mathrm{FF})$ & $\mathrm{AVQZ}^{b}$ & $\mathrm{AVQZ}^{b}$ & 8.52 & 9.58 & -11.00 & 0.05 & 7.15 \\
\hline & & AVQZ & AVTZ-J ${ }^{c}$ & 6.26 & 9.66 & -11.29 & 0.05 & 4.68 \\
\hline
\end{tabular}

$\bar{a}$ The force field basis set is the basis set employed in the cubic force field calculation; the NMR basis set was used for the calculation of the coupling derivatives for the vibrational averaging calculation.

${ }^{b}$ For the SD term the AVTZ basis set was employed.

${ }^{c}$ For the SD term the AVDZ-J basis set was employed.

Table 7 Relativistic corrections to NMR shielding constants in FNNF. Units of ppm

\begin{tabular}{|c|c|c|c|c|c|c|c|c|}
\hline Isomer & $\begin{array}{l}\text { HF } \\
\text { dyall- } \\
\text { cv3z }\end{array}$ & $\begin{array}{r}\text { KT2 } \\
\text { dyall- } \\
\text { cv3z }\end{array}$ & $\begin{array}{r}\text { KT2 } \\
\text { dyall- } \\
\text { acv3z }\end{array}$ & $\begin{array}{r}\text { KT2 } \\
\text { dyall- } \\
\text { acv4z }\end{array}$ & $\begin{array}{r}\text { KT2 } \\
\text { CBS }^{a}\end{array}$ & $\begin{array}{l}\text { PBE0 } \\
\text { dyall- } \\
\text { acv3z }\end{array}$ & $\begin{array}{l}\text { PBE0 } \\
\text { dyall- } \\
\text { acv4z }\end{array}$ & $\begin{array}{l}\mathrm{PBE0} \\
\mathrm{CBS}^{a}\end{array}$ \\
\hline cis & 2.53 & 2.36 & 2.36 & $\begin{array}{r}\sigma(\mathrm{N}) \\
2.35\end{array}$ & 2.35 & 2.37 & 2.35 & 2.34 \\
\hline trans & 2.43 & 2.34 & 2.35 & 2.34 & 2.33 & 2.34 & 2.32 & 2.30 \\
\hline cis & 5.07 & 5.02 & 5.00 & $\begin{array}{r}\sigma(\mathrm{F}) \\
5.00\end{array}$ & 5.00 & 5.01 & 4.98 & 4.96 \\
\hline trans & 4.76 & 4.99 & 4.97 & 4.97 & 4.97 & 4.93 & 4.94 & 4.94 \\
\hline
\end{tabular}

${ }^{a}$ Estimated from acv $(3,4) \mathrm{z}$ results.

Table 8 Relativistic corrections to the isotropic spin-spin coupling constants in FNNF. ${ }^{a}$ Units of $\mathrm{Hz}$

\begin{tabular}{llcccc}
\hline \hline Isomer & Coupling & HF & BP86 & PBE & PBE0-50 \\
\hline \hline cis & IJ(NN) & 0.03 & -0.07 & -0.07 & -0.01 \\
& $1 J(\mathrm{NF})$ & 0.42 & -0.33 & -0.30 & 0.10 \\
& ${ }^{2} J(\mathrm{NF})$ & -0.63 & -0.40 & -0.43 & -0.50 \\
& ${ }^{3} J(\mathrm{FF})$ & 3.23 & 2.88 & 3.09 & 2.24 \\
& & & & & \\
trans & $1 J(\mathrm{NN})$ & -0.21 & -0.30 & -0.30 & -0.21 \\
& $1 J(\mathrm{NF})$ & 0.40 & -0.18 & -0.16 & 0.13 \\
& ${ }^{2} J(\mathrm{NF})$ & -0.50 & -0.13 & -0.15 & -0.33 \\
& ${ }^{3} J(\mathrm{FF})$ & 2.46 & 3.36 & 3.57 & 1.28 \\
\hline \hline
\end{tabular}

${ }^{a}$ Calculated using dyall-acv4z basis set. 
Table 9 Final calculated isotropic shielding constants in FNNF and the comparison with the available literature values. Units of ppm

\begin{tabular}{|c|c|c|c|c|c|c|c|}
\hline \multirow[b]{2}{*}{ Isomer } & \multicolumn{4}{|c|}{ Component $^{a}$} & \multirow[b]{2}{*}{ Total } & \multirow[b]{2}{*}{ Expt } & \multirow[b]{2}{*}{$\mathrm{MCSCF}^{b}$} \\
\hline & Equil. & $\Delta \mathrm{Vib}$ & $\Delta$ Temp & $\Delta$ Rel & & & \\
\hline cis & -137.63 & -5.16 & -0.12 & 2.35 & -140.56 & $-139.25^{c}$ & -110.1 \\
\hline trans & -197.61 & -6.07 & -0.61 & 2.33 & -201.96 & $-201.15^{d}$ & -174.1 \\
\hline cis & 89.86 & -17.46 & -1.81 & 5.00 & 75.59 & $59.92^{e}$ & 137.4 \\
\hline trans & 126.93 & -16.58 & -2.00 & 4.97 & 113.32 & $102.25^{f}$ & 159.9 \\
\hline
\end{tabular}

a Our best estimates: optimized CCSD(T)/ACVQZ equilibrium geometry CBS values (details in Table 3); vibrational corrections (details in Table 5), relativistic corrections (details in Table 7).

${ }^{b}$ RAS-E results. 18

${ }^{c}$ Using $\sigma_{\text {ref }}\left(\mathrm{N}\right.$ in $\left.\mathrm{NF}_{3}\right)=-125.95 \mathrm{ppm}^{15}$ and corresponding $\delta_{\text {exp }}(\mathrm{N})$ of $13.3 \mathrm{ppm} .77$

${ }^{d}$ Using $\sigma_{r e f}\left(\mathrm{~N}^{c}\right.$ in $\left.\mathrm{NF}_{3}\right)=-125.95 \mathrm{ppm}\left[15\right.$ and corresponding $\delta_{\text {exp }}(\mathrm{N})$ of $75.2 \mathrm{ppm} .77$

${ }^{e}$ Using $\sigma_{\text {ref }}\left(\mathrm{F}\right.$ in $\left.\mathrm{CFCl}_{3}\right)=197.07 \mathrm{ppm} \frac{15}{15}$ and corresponding $\delta_{\exp }(\mathrm{F})$ of $137.15 \mathrm{ppm} .78$

${ }^{f}$ Using $\sigma_{\text {ref }}\left(\mathrm{F}_{\text {in }} \mathrm{CFCl}_{3}\right)=197.07 \mathrm{ppm}^{15}$ and corresponding $\delta_{\text {exp }}(\mathrm{F})$ of $94.82 \mathrm{ppm} .78$

Table 10 Final calculated isotropic coupling constants in FNNF and comparison with available literature values. Units of $\mathrm{Hz}$

\begin{tabular}{|c|c|c|c|c|c|c|c|c|}
\hline \multirow[b]{2}{*}{ Isomer } & \multirow[b]{2}{*}{ Coupling } & \multicolumn{2}{|c|}{ Contribution $^{a}$} & \multirow[b]{2}{*}{$\begin{array}{r}\Delta V i b \\
(300 \mathrm{~K}) \\
\end{array}$} & \multirow[b]{2}{*}{$\Delta$ Rel } & \multirow[b]{2}{*}{ Total } & \multirow[b]{2}{*}{$\begin{array}{c}\text { Expt } \\
\text { ref }[78\end{array}$} & \multirow[b]{2}{*}{$\begin{array}{r}\mathrm{MCSCF}^{b} \\
\operatorname{ref} 18\end{array}$} \\
\hline & & $\begin{array}{l}\text { Equil. } \\
\text { ref } 19\end{array}$ & $\Delta \mathrm{CC} 3$ & & & & & \\
\hline \multirow[t]{4}{*}{ cis } & ${ }^{1} J(\mathrm{NN})$ & -0.08 & 1.49 & 0.65 & -0.01 & 2.06 & 4.0 & 3.9 \\
\hline & ${ }^{1} J(\mathrm{NF})$ & 212.32 & 3.50 & -7.09 & 0.01 & 208.75 & 211.0 & 220.8 \\
\hline & ${ }^{2} J(\mathrm{NF})$ & -14.54 & -5.93 & -0.82 & -0.50 & -21.79 & -25.4 & -23.8 \\
\hline & ${ }^{3} J(\mathrm{FF})$ & -127.48 & 3.30 & -4.88 & 2.24 & -126.82 & -146.0 & -131.4 \\
\hline \multirow[t]{4}{*}{ trans } & ${ }^{1} J(\mathrm{NN})$ & -17.53 & -0.22 & -0.29 & -0.21 & -18.25 & -18.5 & $\overline{-12.2}$ \\
\hline & ${ }^{1} J(\mathrm{NF})$ & 177.58 & 1.94 & -6.67 & 0.13 & 172.98 & 172.8 & 172.8 \\
\hline & ${ }^{2} J(\mathrm{NF})$ & -58.66 & -3.11 & 0.12 & -0.33 & -61.97 & -62.8 & -63.4 \\
\hline & ${ }^{3} J(\mathrm{FF})$ & -301.83 & -7.74 & 4.68 & 1.28 & -303.61 & -316.4 & -266.9 \\
\hline
\end{tabular}

a Our best estimates: optimized CCSD(T)/ACVQZ equilibrium geometry values at the CCSD/AV5Z-Juc level from ref [19] CC3 corrections (details in Table 4), vibrational corrections (details in Table 6), relativistic corrections (details in Table 8).

${ }^{b}$ RAS-E approach, ${ }^{14} \mathrm{~N}$ data recomputed to ${ }^{15} \mathrm{~N}$. 